\title{
COMMENT
}

\section{Bringing research directly to families in the era of COVID-19}

\author{
Stephen J. Balevic ${ }^{1,2}$, Lindsay Singler ${ }^{2}$, Rachel Randell ${ }^{1}$, Richard J. Chung ${ }^{1}$, Monica E. Lemmon ${ }^{1,3}$ and Christoph P. Hornik $^{1,2}$ \\ Pediatric Research (2021) 89:404-406; https://doi.org/10.1038/s41390-020-01260-1
}

\section{INTRODUCTION}

The Coronavirus disease 2019 (COVID-19) pandemic is profoundly disrupting clinical research. ${ }^{1}$ In addition to the diversion of research resources towards the pandemic response, resulting in the halting of over 1700 clinical trials, ${ }^{2}$ most ongoing studies have undergone substantial modifications to mitigate infection risk for participants, research staff, and investigators. ${ }^{1}$ Furthermore, the rapid deployment of telemedicine modalities in lieu of face-toface services disrupts typical recruitment methods. The pandemic clearly demonstrates that "brick-and-mortar" site-based research is exquisitely vulnerable to crises that limit access to clinical and research facilities.

By exposing these vulnerabilities, the pandemic also creates opportunities for innovative trial designs. One such example is the decentralized, direct-to-family study design, in which recruitment, intervention, and data collection all occur remotely (e.g., in participants' homes). ${ }^{3-5}$ Remote data collection methods can eliminate the need for site visits, resulting in trials that are more efficient and cost effective. ${ }^{6,7}$ Direct-to-family approaches can recruit larger and more diverse participants compared to traditional trials. ${ }^{6,8}$ An additional advantage during pandemics is the ability to mitigate infectious risk by reducing or eliminating the number of face-to-face encounters between participants and study staff, and avoiding exposure to other patients in the clinic or hospital. Underscoring the benefits of direct-to-family approaches, the US Food and Drug Administration (FDA) recommended virtual assessment and local biosample collection when possible in its guidance for conducting clinical trials during the pandemic. ${ }^{9}$ Further, leaders from regulatory bodies including the Western Institutional Review Board have advocated the use of remote data collection. ${ }^{10}$

The pandemic accentuates existing trends in the pediatric ambulatory healthcare landscape, with increasing consumerism among families creating uncertainty about the future of current care models. ${ }^{11}$ Direct-to-family research is an unprecedented opportunity to respond to these evolutions in pediatric care and increase access to clinical trials during the COVID-19 pandemic and beyond. Herein we share key principles to facilitate the planning, design, and implementation of direct-to-family research studies.

\section{ENSURING PARTICIPANT SAFETY}

The first step in designing a direct-to-family trial is considering the overall risk and potential benefits to participants, and how adequate follow-up and safety can be monitored remotely. An intervention that has an adverse event profile that can be assessed entirely by laboratory data (e.g., elevated liver function tests) would be more fitting for a direct-to-family study than an intervention that could result in clinical signs and symptoms that require a physical examination or interpretation of other clinical data (e.g., shortness of breath or palpitations). Due to the lack of direct clinician contact, most direct-to-family studies in children may be better suited for minimal risk interventions or interventions where a medical product/device has established dosing and/ or safety in a similar population. Earlier phase studies, invasive interventions, and studies requiring investigational new drug applications (IND) that require greater oversight may be less appropriate.

Current regulatory guidance is built on the premise that licensed, and in-state clinicians are available to qualify a safety event; this safeguard may require partnership with out-of-state clinicians in a multi-state direct-to-family trial. Investigators must seek early feedback on study design from local institutional review boards and FDA (in cases of an IND) to guide these and other considerations. Moving forward, policymakers and stakeholders must work together to delineate how direct-to-family studies are best defined, implemented, and regulated. Sponsors, investigators, and regulatory bodies should leverage lessons learned during the COVID-19 pandemic to articulate best practices in remote assessment and safety monitoring.

\section{UNDERSTANDING LEGAL CONSIDERATIONS}

Direct-to-family trials can enroll children from across the United States using a single institution as the primary site, raising important legal considerations when principal investigators (PIs) engage in research in states in which they are not licensed. Legal requirements vary by state, but, in general, two-way research, in which participants receive either a diagnosis or other intervention (including an investigational product/device) is typically considered to create a patient-provider relationship. In most cases, this requires that the $\mathrm{PI}$ have medical licensure in the state in which the participant resides. Some states require in-person examination before medications can be prescribed, while other states allow video or other remote assessments to fulfill this requirement. Practically speaking, an interventional, direct-to-family study that recruits children from across multiple states may require partnership with physicians in other states, or the PI could consider obtaining multi-state licensure. Investigators should confirm general liability and malpractice coverage in all cases, especially when there is physical interaction with a participant.

In general, one-way research in which a participant does not receive a diagnosis or treatment/intervention through the study does not require a PI to have an in-state license. Investigators can further mitigate the perception of a patient-provider relationship by explicitly stating in the protocol and consent forms that the study is for research purposes only. However, some states may

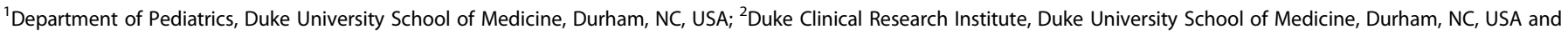
${ }^{3}$ Department of Population Health Sciences, Duke University School of Medicine, Durham, NC, USA
}

Correspondence: Christoph P. Hornik (Christoph.hornik@duke.edu)

Received: 9 October 2020 Accepted: 16 October 2020

Published online: 11 November 2020 
require home healthcare personnel (e.g., mobile research nurses) to operate under the license of an in-state physician, even in oneway research studies. The complexity and variability of state legal requirements pose a barrier to the widespread implementation of direct-to-family studies. Stakeholders should advocate for streamlined processes to facilitate the conduct of direct-to-family research, investment in infrastructure to support multi-state research teams, re-examination of licensure requirements for low-risk direct-to-family research studies, and standardization of state requirements.

\section{LEVERAGING TECHNOLOGY}

Technology is a key underpinning of direct-to-family trials, with advances in wearable devices and other electronic platforms (e.g., surveys, telemedicine devices) enabling collection of a wide variety of data from participants' homes. In addition, blood or other biosamples can be obtained through either participant selfcollection or mobile phlebotomy. ${ }^{12,13}$ These technologies offer an opportunity to collect novel forms of data in real-world environments, offering the potential to improve the reliability, generalizability, and applicability of study findings. ${ }^{14}$

The direct-to-family design is well-suited to leverage emerging technology, but defining remote outcome measures is a critical priority for the field. Many outcome measures require in-person assessment; for example, a therapeutic trial in juvenile arthritis may require a hands-on joint exam. In contrast, assessing patientreported outcomes that do not rely on physical exam features are amenable to remote data collection. While many traditional outcome measures used in children can be modified for remote assessment, investigators should carefully consider whether such changes require further validation. The research community should work to develop and validate outcome measures that can be administered remotely. Regulatory bodies should support the inclusion of evidence-based remote assessments as clinical trial endpoints.

\section{FACILITATING STAKEHOLDER ENGAGEMENT}

Stakeholder perspectives are critical in increasing participant and caregiver engagement and retention. Involving stakeholders, such as patient groups, in the research process can improve the quality and efficiency of research; help researchers better understand illness experiences, burdens, preferences, risk tradeoffs, and barriers; and accelerate the translation of research findings into clinical practice. ${ }^{15}$ Assessing the caregiver perspective on key features of direct-to-family design, such as remote assessments, biospecimen collection, nursing visits, and the use of technology, is critical. Through focus groups, stakeholder advisory boards, and other models of engagement, investigators can engage participants and their caregivers as partners throughout the research process, from development of the study concept and design to dissemination of research results.

Direct-to-family designs require investigators to build trust and retain participants without on-site visits. Pediatric participants and their caregivers value the involvement of their primary clinicians in any direct-to-family research model to support their participation, communicate treatment changes, and perform endpoint assessments. Investigators could consider engaging participants' primary clinicians by having a clinician-facing explanation of the study that potential participants could share during the recruitment process. Pediatric patients and their caregivers also endorse the critical role of a steady point of contact, such as a study concierge, in the direct-to-family research model. This role can help build trust and familiarity with the study, and enhance adherence to study procedures and overall retention. Investigators should consider key family-facing personnel who can play this role outside of a research site or clinic. Finally, incentive structures that rely on behavioral economic principles, including financial incentives, may be uniquely suited to enhance retention in the absence of in-person contact.

\section{CONCLUSION}

Direct-to-family study designs are patient- and family-centric, leveraging technology to safely and efficiently conduct clinical research in the comfort of a participant's home. In order to optimize direct-to-family trials, investigators should seek early feedback from regulatory authorities about proposed safety and follow-up procedures, and feedback from family and other stakeholders on study schedules and interventions. Pediatric clinical research must adapt to be more flexible, efficient, and cost effective to increase access to clinical trials both during the COVID-19 pandemic and beyond.

\section{ACKNOWLEDGEMENTS}

Experiences and expertise discussed in this commentary were gathered through the design of a direct to families trial support by the U.S. Food and Drug Administration supported Global Pediatric Clinical Trials Network (U18-FD006298).

\section{AUTHOR CONTRIBUTIONS}

All authors meet the criteria for authorship and contributed to the conception and design, acquisition of data, or analysis and interpretation of data; and drafted the article or revised it critically for important intellectual content. All authors approved the final version for submission.

\section{ADDITIONAL INFORMATION}

Competing interests: S.J.B. receives support from the National Institutes of Health FDA, PCORI, the Rheumatology Research Foundation's Scientist Development Award, the Childhood Arthritis and Rheumatology Research Alliance, and consulting for UCB. L.S.'s authorship of this manuscript was supported by the U.S. Food and Drug Administration under the Global Pediatric Clinical Trials Network, Award Number 5U18FD006298. R.R. receives support from the National Institute of General Medical Sciences and the Eunice Kennedy Shriver National Institute of Child Health \& Human Development of the National Institutes of Health under Award Number T32GM086330. R.R.'s spouse has current or prior employment and/or stock ownership in Merck \& Co. and Biogen. R.J.C. has no disclosures to report. M.E.L. receives salary support from the National Institute of Neurological Disorders and Stroke of the National Institutes of Health under Award Number (K23NS116453). C.P. $\mathrm{H}$. receives salary support for research from National Institute for Child Health and Human Development (NICHD) (R13HD102136), the National Heart Lung and Blood Institute (NHLBI) (R61/R33HL147833), the US Food and Drug Administration (R01FD006099, PI Laughon; and U18-FD006298, PI Benjamin), the U.S. government for his work in pediatric clinical pharmacology (Government Contract HHSN275201800003I, PI: Benjamin under the Best Pharmaceuticals for Children Act), the non-profit Burroughs Wellcome Fund, and other sponsors for drug development in adults and children (https://dcri.org/about-us/conflict-of-interest/).

Publisher's note Springer Nature remains neutral with regard to jurisdictional claims in published maps and institutional affiliations.

\section{REFERENCES}

1. Weiner, D. L., Balasubramaniam, V., Shah, S. I. \& Javier, J. R. COVID-19 impact on research, lessons learned from COVID-19 research, implications for pediatric research. Pediatr. Res. 88, 148-150 (2020).

2. Carlisle, B. G. Clinical trials stopped by Covid-19. https://covid19.bgcarlisle.com/. Accessed 28 Sep 2020 (2020).

3. Marquis-Gravel, G. et al. Rationale and Design of the Aspirin Dosing-A PatientCentric Trial Assessing Benefits and Long-term Effectiveness (ADAPTABLE) Trial. JAMA Cardiol. 5, 598-607 (2020).

4. Orri, M., Lipset, C. H., Jacobs, B. P., Costello, A. J. \& Cummings, S. R. Web-based trial to evaluate the efficacy and safety of tolterodine ER $4 \mathrm{mg}$ in participants with overactive bladder: REMOTE trial. Contemp. Clin. Trials 38, 190-197 (2014).

5. Sommer, C. et al. Building clinical trials around patients: evaluation and comparison of decentralized and conventional site models in patients with low back pain. Contemp. Clin. Trials Commun. 11, 20-26 (2018).

6. Ali, Z., Zibert, J. R. \& Thomsen, S. F. Virtual clinical trials: perspectives in dermatology. Dermatology 236, 375-382 (2020). 
7. Shore, C., Khandekar, E. \& Alper, J. Virtual Clinical Trials: Challenges and Opportunities: Proceedings of a Workshop (National Academies Press: Washington, DC, 2019).

8. Rosa, C., Campbell, A. N., Miele, G. M., Brunner, M. \& Winstanley, E. L. Using etechnologies in clinical trials. Contemp. Clin. Trials 45, 41-54 (2015).

9. FDA Guidance on Conduct of Clinical Trials of Medical Products during the COVID-19 Public Health Emergency. Guidance for Industry, Investigators, and Institutional Review Boards. 1-35 (Administration UDoHaHSFaD, 2020).

10. Young, M. IRB Experts Offer Advice for Changing Research Landscape [cited 9/23/2020]. https://www.reliasmedia.com/articles/146297-irb-experts-offeradvice-for-changing-research-landscape. Accessed 28 Sep 2020 (2020).

11. Ray, K. N. et al. Trends in pediatric primary care visits among commercially insured US children, 2008-2016. JAMA Pediatr. 174, 350-357 (2020).
12. Hansen, T. V., Simonsen, M. K., Nielsen, F. C. \& Hundrup, Y. A. Collection of blood, saliva, and buccal cell samples in a pilot study on the Danish nurse cohort: comparison of the response rate and quality of genomic DNA. Cancer Epidemiol. Biomark. Prev. 16, 2072-2076 (2007).

13. Randell, R. L. et al. Collecting biospecimens from an internet-based prospective cohort study of inflammatory bowel disease (CCFA Partners): a feasibility study. JMIR Res. Protoc. 5, e3 (2016).

14. Khozin, S. \& Coravos, A. Decentralized trials in the age of real-world evidence and inclusivity in clinical investigations. Clin. Pharm. Ther. 106, 25-27 (2019).

15. Levitan, B., Getz, K. \& Eisenstein, E. L. Assessing the financial value of patient engagement: a quantitative approach from CTTl's patient groups and clinical trials project. Ther. Innov. Regul. Sci. 52, 220-229 (2018). 